\title{
POINTWISE ESTIMATES FOR AN INTERPOLATION PROCESS OF S. N. BERNSTEIN
}

\author{
A. K. VARMA and XIANG MING YU
}

(Received 12 December 1989)

\begin{abstract}
The main object of this paper is to provide the solution of an open problem raised by Professor Ron DeVore concerning constructing interpolating process $H_{n}[f, x]$ satisfying the inequality (1.11). Results on simultaneous approximation are also obtained.
\end{abstract}

1980 Mathematics subject classification (Amer. Math. Soc.) (1985 Revision): 42 A 15.

Keywords and phrases: rate of convergence, interpolation process.

\section{Introduction and main results}

Jackson's well-known theorem [6] concerning best approximation is of fundamental importance in the constructive theory of functions. Subsequently, Jackson's theorem has been sharpened by S. N. Nikolskii [10], A. F. Timan [12]. The essential feature of Timan's theorem is the improvement of the order of approximation near the ends of the interval.

For a function $f(x)$ defined on $[-1,1]$ we put

$$
\omega_{r}(f, t)=\sup _{-1 \leq x, x+h r \leq 1|h| \leq t} \sup _{h}\left|\Delta_{h}^{r} f(x)\right|
$$

for the $r$ th modulus of smoothness. Timan's theorem has been generalized by J. A. Brudnyi [1] and may be stated as follows.

THEOREM A. For every continuous function $f(x)$ defined on $[-1,1]$ and for an arbitrary natural number $r$ and $n$ greater than or equal to $r-1$

(C) 1991 Australian Mathematical Society $0263-6115 / 91 \$ A 2.00+0.00$ 
there exists an algebraic polynomial $P_{n}(x)$ of degree at most $n$ such that, for $-1 \leq x \leq 1$,

$$
\left|f(x)-P_{n}(x)\right| \leq A_{r} \omega_{r}\left(f,\left(\frac{\left(1-x^{2}\right)^{1 / 2}}{n}\right)+\left(\frac{1}{n}\right)^{2}\right)
$$

where $A_{r}$ is a constant depending only on $r$.

In an earlier work the first author [13] gave a new proof of the inequality (1.2) for the case $r=1$. Here the process is a weakly interpolatory in the sense that it is uniquely determined by the values of the given function at the zeros of Tchebycheff polynomial of the first kind.

There is an interesting question that was posed both by G. G. Lorentz and S. B. Steckin as to whether it is possible to replace the inequality (1.2) by

$$
\left|f(x)-P_{n}(x)\right| \leq A_{r} \omega_{r}\left(f, \frac{\sqrt{1-x^{2}}}{n}\right) .
$$

This was shown to be possible in the case $r=1$ by S. Teljakovskii [11] and in the case $r=2$ by Ron DeVore [5]. The first author and T. M. Mills [8] also gave an interpolatory proof of the inequality (1.3) for $r=1$. It turns out the process developed in [8] cannot provide the proof of inequality (1.3) for $r=2$.

In a letter to the first author, Ron DeVore raised the problem of obtaining an interpolatory process which also provides the inequality for $r=2$ as well. The main object of this paper is to show that the answer to this interesting question is in the affirmative. Now we shall turn to describe these results.

Let

$$
T_{n}(x)=\cos n \theta, \cos \theta=x,-1 \leq x \leq 1,
$$

be the Tchebycheff polynomial of degree $n$. We denote by

$$
l_{k n}(x)=\frac{(-1)^{k+1}\left(1-x_{k n}^{2}\right)^{1 / 2}}{n} \frac{T_{n}(x)}{x-x_{k n}}, k=1,2, \ldots, n
$$

the fundamental polynomials of Lagrange interpolation based on the nodes $x_{k n}$ where

$$
x_{k n}=\cos \frac{(2 k-1) \pi}{2 n}, k=1,2, \ldots, n
$$


are the zeros of $T_{n}(x)$ in $(-1,1)$. Write

$$
\left\{\begin{array}{l}
\phi_{1 n}(x)=\left(\frac{3 l_{1 n}(x)+l_{2 n}(x)}{4}\right), \phi_{n, n}(x)=\left(\frac{l_{n-1, n}(x)+3 l_{n n}(x)}{4}\right), \\
\phi_{k n}(x)=\left(\frac{l_{k-1, n}(x)+2 l_{k n}(x)+l_{k+1, n}(x)}{4}\right), k=2,3, \ldots, n-1,
\end{array}\right.
$$

and

$$
\left\{\begin{array}{l}
\chi_{1, n}(x)=\left(\frac{3 \phi_{1 n}(x)+\phi_{2, n}(x)}{4}\right), \chi_{n, n}(x)=\left(\frac{\phi_{n-1, n}(x)+3 \phi_{n n}(x)}{4}\right), \\
\chi_{k n}(x)=\left(\frac{\phi_{k-1, n}(x)+2 \phi_{k n}(x)+\phi_{k+1, n}(x)}{4}\right), k=2,3, \ldots, n-1 .
\end{array}\right.
$$

Let $f \in C[-1,1]$, we define

$$
G_{n}(f, x)=\sum_{k=1}^{n} f\left(x_{k n}\right) \chi_{k n}(x)
$$

and

$$
\begin{aligned}
H_{n}(f, x)= & G_{n}(f, x)-\frac{(1+x)}{2}\left(G_{n}(f, 1)-f(1)\right) \\
& -\frac{(1-x)}{2}\left(G_{n}(f,-1)-f(-1)\right) .
\end{aligned}
$$

Concerning $H_{n}(f, x)$ we shall prove the following.

Theorem 1. Let $f \in C[-1,1]$ and $H_{n}[f, x]$ be defined as in (1.10). Then we have

$$
\left|f(x)-H_{n}(f, x)\right| \leq C_{1} \omega_{2}\left(f ; \frac{\sqrt{1-x^{2}}}{n}\right) .
$$

TheOREM 2. Let $f \in C^{(1)}[-1,1]$ and $H_{n}[f, x]$ be defined as in (1.10). Then we have

$$
\left|f^{\prime}(x)-H_{n}^{\prime}(f, x)\right| \leq c_{2} \omega\left(f^{\prime} ;\left(\frac{\sqrt{1-x^{2}}}{n}\right)+\frac{1}{n^{2}}\right) .
$$

Furthermore if

$$
\begin{aligned}
Q_{n}^{\prime}(f, x)=H_{n}^{\prime}(f, x) & -\frac{1+x}{2}\left[H_{n}^{\prime}(f, 1)-f^{\prime}(1)\right] \\
& -\frac{(1-x)}{2}\left[H_{n}^{\prime}(f,-1)-f^{\prime}(-1)\right]
\end{aligned}
$$


then

$$
\left|Q_{n}^{\prime}(f, x)-f^{\prime}(x)\right| \leq c_{3} \omega\left(f^{\prime} ; \frac{\sqrt{1-x^{2}}}{n}\right)
$$

The next theorem provides the solution of the problem of simultaneous approximation of a function and its derivatives through interpolation polynomials (weak interpolation).

THEOREM 3. Let $f(x) \in C^{(1)}[-1,1]$ and

$$
\begin{aligned}
J_{n}(f, x)= & H_{n}(f, x)-\frac{(1+x)^{2}}{4 n^{2}}\left(T_{n}(x)-T_{n}(1)\right)\left(H_{n}^{\prime}(f, 1)-f^{\prime}(1)\right) \\
& -\frac{(1-x)^{2}(-1)^{n+1}}{4 n^{2}}\left(T_{n}(x)-T_{n}(-1)\right)\left(H_{n}^{\prime}(f,-1)-f^{\prime}(-1)\right) .
\end{aligned}
$$

Then we have

$$
\left|f^{(r)}(x)-J_{n}^{(r)}(f, x)\right| \leq c \frac{\sqrt{1-x^{2}}}{n} \omega\left(f^{\prime}, \frac{\sqrt{1-x^{2}}}{n}\right), r=0,1
$$

\section{Representation problem}

Let $\pi_{n}$ be the set of all polynomials of degree at most $n$. We shall prove the following formula for $P_{n-1} \in \pi_{n-1}$.

$$
\text { LeMma 2.1. Let } P_{n-1} \in \pi_{n-1} \text {. Then we have }(x=\cos \theta)
$$

$$
\begin{aligned}
& G_{n}\left(P_{n-1}, x\right) \\
& =\frac{1}{16}\left\{P_{n-1}(\cos (\theta+2 \pi / n))+4 P_{n-1}(\cos (\theta+\pi / n)\right. \\
& \left.\quad+6 P_{n-1}(\cos \theta)+4 P_{n-1}(\cos (\theta-\pi / n))+P_{n-1}(\cos (\theta-2 \pi / n))\right\} .
\end{aligned}
$$

Since $G_{n}(f, x)$ is a linear process, we only need to verify if $(2.1)$ is valid for

$$
P_{n-1}(x)=\cos \nu \theta, \quad \nu=0,1, \ldots, n-1 .
$$


From the definition of $G_{n}(f, x)$ we have

$$
G_{n}\left(P_{n-1}, x_{j n}\right)=\sum_{k=1}^{n} \cos \nu \theta_{k n} \chi_{k n}\left(x_{j n}\right) .
$$

From (1.7) and (1.8) we have

$$
\begin{aligned}
\chi_{1 n}(x) & =\frac{10 l_{1 n}(x)+5 l_{2 n}(x)+l_{3, n}(x)}{16}, \\
\chi_{2 n}(x) & =\left(5 l_{1 n}(x)+6 l_{2 n}(x)+4 l_{3 n}(x)+l_{4, n}(x)\right) 16, \\
\chi_{k n}(n) & =\frac{l_{k-2, n}(x)+4 l_{k-1, n}(x)+6 l_{k n}(x)+4 l_{k+1, n}(x)+l_{k+2, n}(x)}{16}, k=3,4, \ldots, n-2, \\
\chi_{n-1, n}(x) & =\frac{5 l_{n, n}(x)+6 l_{n-1, n}(x)+4 l_{n-2, n}(x)+l_{n-3, n}(x)}{16}, \\
\chi_{n, n}(x) & =\frac{10 l_{n n}(x)+5 l_{n-1, n}(x)+l_{n-2, n}(x)}{16} .
\end{aligned}
$$

Denote by $I_{n}\left(P_{n-1}, x\right)$ the right hand side of (2.1). For $j=1$ we have

$$
\begin{aligned}
\left(P_{n-1}(x)\right. & =\cos \nu \theta, \cos \theta=x) G_{n}\left(P_{n-1}, x_{1 n}\right) \\
& =(10 / 16) \cos \nu \theta_{1 n}+(5 / 16) \cos \nu \theta_{2 n}+(1 / 16) \cos \nu \theta_{3 n}
\end{aligned}
$$

and

$$
\begin{aligned}
I_{n}\left(P_{n-1}, x_{1 n}\right)= & \frac{1}{16}\left[\cos \nu\left(\theta_{1}+\frac{2 \pi}{n}\right)+4 \cos \nu\left(\theta_{1 n}+\frac{\pi}{n}\right)+4 \cos \nu\left(\theta_{1 n}+\frac{\pi}{n}\right)\right. \\
& \left.+6 \cos \nu \theta_{1 n}+4 \cos \nu\left(\theta_{1 n}-\frac{\pi}{n}\right)+\cos \nu\left(\theta_{1 n}-\frac{2 \pi}{n}\right)\right] \\
= & \frac{1}{16}\left[10 \cos \frac{\nu \pi}{2 n}+5 \cos \nu \frac{3 \pi}{2 n}+\cos \nu \frac{5 \pi}{2 n}\right] \\
= & G_{n}\left(P_{n-1}, x_{1 n}\right) .
\end{aligned}
$$

Similarly, we can prove that $G_{n}\left(P_{n-1}, x_{j n}\right)=I_{n}\left(P_{n-1}, x_{j n}\right)$ for $j=$ $2,3, \ldots, n$. Now we note that $G_{n}\left(P_{n-1}, x\right)$ and $I_{n}\left(P_{n-1}, x\right)$ are both polynomials of degree at most $n-1$ and agree with each other at $x=x_{j n}$, $j=1,2, \ldots, n$. From this it follows that

$$
G_{n}\left(P_{n-1}, x\right) \equiv I_{n}\left(P_{n-1}, x\right)
$$

and the lemma is proved. 


\section{Estimates of $A_{n}(f, x)$}

Put

$$
\begin{aligned}
A_{n}(f, x)= & 2\left[f\left(x \cos \frac{2 \pi}{n}\right)-f(x)\right]+8\left[f\left(x \cos \frac{\pi}{n}\right)-f(x)\right] \\
& -(1+x)\left[f\left(\cos \frac{2 \pi}{n}\right)+4 f\left(\cos \frac{\pi}{n}\right)-5 f(1)\right] \\
& -(1-x)\left[f\left(-\cos \frac{2 \pi}{n}\right)+4 f\left(-c \cos \frac{\pi}{n}\right)-5 f(-1)\right] .
\end{aligned}
$$

We shall now prove the following.

LEMMA 3.1. Let $f \in C^{(2)}[-1,1]$ then we have

$$
\left|A_{n}(f, x)\right| \leq c \frac{\left(1-x^{2}\right)}{n^{2}} M_{2}, M_{2}=\max _{-1 \leq x \leq 1}\left|f^{\prime \prime}(x)\right|,
$$

where $c$ is an absolute constant independent of $n, x$ and $f$.

Proof. A simple calculation shows that

$$
\begin{aligned}
& f\left(\cos \frac{2 \pi}{n}\right)+4 f\left(\cos \frac{\pi}{n}\right)-5 f(1) \\
& \quad=\left(\cos \frac{2 \pi}{n}-1\right) f^{\prime}\left(\eta_{1}\right)+4\left(\cos \frac{\pi}{n}-1\right) f^{\prime}\left(\eta_{2}\right)
\end{aligned}
$$

and

$$
\begin{aligned}
& f\left(-\cos \frac{2 \pi}{n}\right)+4 f\left(-\cos \frac{\pi}{n}\right)-5 f(-1) \\
& \quad=\left(1-\cos \frac{2 \pi}{n}\right) f^{\prime}\left(\eta_{3}\right)+4\left(1-\cos \frac{\pi}{n}\right) f^{\prime}\left(\eta_{4}\right)
\end{aligned}
$$

where $-1<\eta_{1}, \eta_{2}, \eta_{3}, \eta_{4},<1$. Also note that

$$
A_{n}(f, \pm 1)=0
$$


From (3.3), (3.4) and (3.1) we have

$$
\begin{aligned}
A_{n}^{\prime}(f, x)= & 2\left[\cos \left(\frac{2 \pi}{n}\right) f^{\prime}\left(x \cos \frac{2 \pi}{n}\right)-f^{\prime}(x)\right] \\
& +8\left[\cos \frac{\pi}{n} f^{\prime}\left(x \cos \frac{\pi}{n}\right)-f^{\prime}(x)\right] \\
& -\left(\cos \frac{2 \pi}{n}-1\right) f^{\prime}\left(\eta_{1}\right)-4\left(\cos \frac{\pi}{n}-1\right) f^{\prime}\left(\eta_{2}\right) \\
& +\left(1-\cos \frac{2 \pi}{n}\right) f^{\prime}\left(\eta_{3}\right)+4\left(1-\cos \frac{\pi}{n}\right) f^{\prime}\left(\eta_{4}\right) \\
= & 2\left[\cos \frac{2 \pi}{n}\left(f^{\prime}\left(x \cos \frac{2 \pi}{n}\right)-f^{\prime}(x)\right)+f^{\prime}(x)\left(\cos \frac{2 \pi}{n}-1\right)\right] \\
& +8\left[\cos \frac{\pi}{n}\left(f^{\prime}\left(x \cos \frac{\pi}{n}\right)-f^{\prime}(x)\right)+f^{\prime}(x)\left(\cos \frac{\pi}{n}-1\right)\right] \\
& -\left(\cos \frac{2 \pi}{n}-1\right) f^{\prime}\left(\eta_{1}\right)-4\left(\cos \frac{\pi}{n}-1\right) f^{\prime}\left(\eta_{2}\right) \\
& +\left(1-\cos \frac{2 \pi}{n}\right) f^{\prime}\left(\eta_{3}\right)+4\left(1-\cos \frac{\pi}{n}\right) f^{\prime}\left(\eta_{4}\right) \\
= & 2 x \cos \frac{2 \pi}{n}\left(\cos \frac{2 \pi}{n}-1\right) f^{\prime \prime}\left(\eta_{5}\right)+8 x \cos \frac{\pi}{n}\left(\cos \frac{\pi}{n}-1\right) f^{\prime \prime}\left(\eta_{6}\right) \\
& +\left(\cos \frac{2 \pi}{n}-1\right)\left(2 f^{\prime}(x)-f^{\prime}\left(\eta_{1}\right)-f^{\prime}\left(\eta_{3}\right)\right) \\
& +4\left(\cos \frac{\pi}{n}-1\right)\left(2 f^{\prime}(x)-f^{\prime}\left(\eta_{2}\right)-f^{\prime}\left(\eta_{4}\right)\right) .
\end{aligned}
$$

Therefore, by the Mean Value Theorem we have

$$
\left|A_{n}^{\prime}(f, x)\right| \leq 32 \sin ^{2}\left(\frac{\pi}{n}\right) M_{2} .
$$

Next, let $0 \leq x \leq 1$ then by (3.5) and (3.6) we have

$$
A_{n}(f, x)=A_{n}(f, x)-A_{n}(f, 1)=(x-1) A_{n}^{\prime}(f, \xi) .
$$

Hence

$$
\left|A_{n}(f, x)\right| \leq(1-x) 32 M_{2} \sin ^{2} \frac{\pi}{n} \leq \frac{32 M_{2}\left(1-x^{2}\right) \pi^{2}}{n^{2}} .
$$

Similarly if $-1 \leq x \leq 0$, we obtain

$$
\left|A_{n}(f, x)\right| \leq \frac{32 M_{2}\left(1-x^{2}\right) \pi^{2}}{n^{2}} .
$$

From (3.7) and (3.8) we obtain the required result. 


\section{Main estimates}

We shall now be able to prove the following.

LEMMA 4.1. Let $f \in C^{(2)}[-1,1]$ and let $q_{n-1}(x)$ be the polynomial satisfying the inequality, for $-1 \leq x \leq 1$

$$
\left|f(x)-q_{n-1}(x)\right| \leq c_{1} \frac{1-x^{2}}{n^{2}} M_{2}, M_{2}=\max _{-1 \leq x \leq 1}\left|f^{\prime \prime}(x)\right| .
$$

\section{Then we have}

$$
\left|H_{n}\left[q_{n-1}, x\right]-q_{n-1}(x)\right| \leq c_{2}\left(\Delta_{n}(x)\right)^{2} M_{2},-1 \leq x \leq 1,
$$

where $c_{1}, c_{2}$ are absolute positive constants independent of $n, x, f$, and

$$
\Delta_{n}(x)=\frac{\sqrt{\left(1-x^{2}\right)}}{n}+\left(\frac{1}{n}\right)^{2} .
$$

Proof. From the definition of $H_{n}(f, x)$ as given by (1.9), (1.10) and Lemma 2.1 we have

$$
\begin{aligned}
16\left(H_{n}\left(q_{n-1}, x\right)-q_{n-1}(x)\right)=q_{n-1}\left(\cos \left(\theta+\frac{2 \pi}{n}\right)\right)+4 q_{n-1}\left(\cos \left(\theta+\frac{\pi}{n}\right)\right) \\
\quad+4 q_{n-1}\left(\cos \left(\theta-\frac{\pi}{n}\right)\right)-10 q_{n-1}(\cos \theta)+q_{n-1}\left(\cos \left(\theta-\frac{2 \pi}{n}\right)\right) \\
(4.3) \quad-(1+x)\left\{q_{n-1}\left(\cos \frac{2 \pi}{n}\right)+4 q_{n-1}\left(\cos \frac{\pi}{n}\right)-5 q_{n-1}(1)\right\} \\
\left.\quad-(1-x)\left\{q_{n-1}\left(-\cos \frac{2 \pi}{n}\right)+4 q_{n-1}\left(-\cos \frac{\pi}{n}\right)-5 q_{n-1}(-1)\right)\right\} \\
=A_{n}\left(q_{n-1}, x\right)+B_{n}\left(q_{n-1}, x\right)
\end{aligned}
$$

where $A_{n}\left(q_{n-1}, x\right)$ is defined by $(3.1)$ and $B_{n}\left(q_{n-1}, x\right)$ is stated as follows.

$$
\begin{aligned}
B_{n}\left(q_{n-1}, x\right)= & q_{n-1}\left(\cos \left(\theta+\frac{2 \pi}{n}\right)\right)+q_{n-1}\left(\cos \left(\theta-\frac{2 \pi}{n}\right)\right) \\
& -2 q_{n-1}\left(\cos \theta \cos \frac{2 \pi}{n}\right) \\
+ & 4\left[q_{n-1}\left(\cos \left(\theta+\frac{\pi}{n}\right)\right)+q_{n-1}\left(\cos \left(\theta-\frac{\pi}{n}\right)\right)\right. \\
& \left.-2 q_{n-1}\left(\cos \theta \cos \frac{\pi}{n}\right)\right] .
\end{aligned}
$$

If we set $\alpha_{1}=\cos \theta \cos \frac{2 \pi}{n}, \beta_{1}=\sin \theta \sin \frac{2 \pi}{n}, \alpha_{2}=\cos \theta \cos \frac{\pi}{n}$, and $\beta_{2}=$ $\sin \theta \sin \frac{\pi}{n}$ then

$$
\begin{aligned}
B_{n}\left(q_{n-1}, x\right)= & \Delta_{\beta_{1}}^{2} q_{n-1}\left(\alpha_{1}\right)+\Delta_{\beta_{1}}^{2} q_{n-1}\left(\alpha_{2}\right) \\
= & \Delta_{\beta_{1}}^{2}\left(q_{n-1}\left(\alpha_{1}\right)-f\left(\alpha_{1}\right)\right)+\Delta_{\beta_{1}}^{2}\left(q_{n-1}\left(\alpha_{2}\right)-f\left(\alpha_{2}\right)\right) \\
& +\Delta_{\beta_{1}}^{2} f\left(\alpha_{1}\right)+\Delta_{\beta_{2}}^{2} f\left(\alpha_{2}\right) .
\end{aligned}
$$


Since $\beta_{1}, \beta_{2}=O\left(\frac{\sqrt{1-x^{2}}}{n}\right)$ and $1-\alpha_{1}^{2}=O\left(1-x^{2}+n^{-2}\right)$ and $1-\alpha_{2}^{2}=$ $O\left(1-x^{2}+n^{-2}\right)$ it follows from (4.1) and (4.5) that

$$
B_{n}\left(q_{n-1}, x\right)=O\left(\frac{1-x^{2}}{n^{2}}+\frac{1}{n^{4}}\right) M_{2} .
$$

Furthermore by (4.1) and (3.1) we have

$$
\left|A_{n}\left(q_{n-1}-f, x\right)\right|=O\left(\frac{1-x^{2}}{n^{2}}+\frac{1}{n^{4}}\right) M_{2} .
$$

Next by (4.3), (3.2), (4.6), (4.7) we have

$$
\begin{aligned}
16\left[H_{n}\left(q_{n-1}, x\right)-q_{n-1}(x)\right] & \\
\quad= & A_{n}\left(q_{n}-f, x\right)+A_{n}(f, x)+B_{n}\left(q_{n-1}, x\right) \\
\quad= & O\left(\frac{1-x^{2}}{n^{2}}+\frac{1}{n^{4}}\right) M_{2} .
\end{aligned}
$$

This proves Lemma 4.1 .

We also remark that if $f \in C^{(2)}[-1,1]$ then

(4.9) $\left|H_{n}\left[q_{n-1}, x\right]-q_{n-1}(x)\right|=O\left(\frac{1-x^{2}}{n^{2}}\right) M_{2}, M_{2}=\max _{-1 \leq x \leq 1}\left|f^{\prime \prime}(x)\right|$.

Let $0 \leq x \leq 1$, then

$$
\begin{aligned}
& H_{n}\left[q_{n-1}, x\right]-q_{n-1}(x) \\
& \quad=H_{n}\left[q_{n-1}, x\right]-q_{n-1}(x)-\left(H_{n}\left(q_{n-1}, 1\right)-q_{n-1}(1)\right) \\
& \quad=\left[H_{n}^{\prime}\left[q_{n-1}, \xi\right)-q_{n-1}^{\prime}(\xi)\right](1-x)
\end{aligned}
$$

where $x \leq \xi \leq 1$.

If $\sqrt{1-x} \geq 1 / n$ then from (4.2) it follows that $\left|H_{n}\left[q_{n-1}, x\right]-q_{n-1}(x)\right|=$ $O\left(\frac{1-x^{2}}{n^{2}}\right) M_{2}$. When $\sqrt{1-x}<1 / n$ we use $(4.10)$ and

$$
\left|H_{n}^{\prime}\left[q_{n-1}, x\right]-q_{n-1}^{\prime}(x)\right|=O\left(\frac{1-x^{2}}{n}+\frac{1}{n^{2}}\right) M_{2}=O\left(\frac{1}{n^{2}}\right) M_{2} .
$$

(This is immediate from the result of V. K. Dzyadyk [3] and the fact that $H_{n}\left(q_{n-1}, x\right)-q_{n-1}(x)$ is itself a polynomial of degree at most $n-1$.) Combining the results of (4.10) and (4.11) we have

$$
\left|H_{n}\left[q_{n-1}, x\right]-q_{n-1}(x)\right| \leq C \frac{1-x}{n^{2}} M_{2} \leq C_{1} \frac{1-x^{2}}{n^{2}} M_{2} .
$$

The proof of (4.9) for the case when $-1 \leq x \leq 0$ is similar. 


\section{Fundamental estimates}

Here we shall prove the following.

LeMma 5.1. Let $f \in C^{(2)}[-1,1]$ and let $q_{n-1}(x)$ be the polynomial of degree at most $n-1$ satisfying (4.1). Then we have

$$
\left|H_{n}\left(f-q_{n-1}, x\right)\right| \leq C_{3} \frac{1-x^{2}}{n^{2}} M_{2} .
$$

Proof. First we give a corresponding estimate for $G_{n}(f, x)$. From the definition of $G_{n}(f, x)$ as given by (1.9) we have

$$
G_{n}\left(f-q_{n-1}, x\right)=\sum_{k=1}^{n}\left(f\left(x_{k n}\right)-q_{n-1}\left(x_{k n}\right)\right) \chi_{k n}(x) .
$$

From (4.1) and [13] ( $C$ is an absolute positive constant) we have

$$
\sum_{k=1}^{n}\left|\chi_{k n}(x)\right| \leq C
$$

and it follows that $1-x_{1 n}^{2}=O\left(n^{-2}\right), 1-x_{n n}^{2}=O\left(n^{-2}\right)$,

$$
\left|\left(f\left(x_{k n}\right)-q_{n-1}\left(x_{k n}\right)\right) \chi_{k n}(x)\right|=O\left(n^{-4}\right) M_{2}, k=1,2 ; n-1, n .
$$

Next we consider the sum

$$
c(x)=\sum_{k=3}^{n-2}\left(f\left(x_{k n}\right)-q_{n-1}\left(x_{k n}\right)\right) \chi_{k n}(x) .
$$

Clearly

$$
\begin{aligned}
&|c(x)| \leq \frac{C M_{2}}{n^{2}} \sum_{k=3}^{n-2}\left(1-x_{k n}^{2}\right)\left|\chi_{k n}(x)\right| \\
& \leq \frac{C M_{2}}{4 n^{2}}\left\{\sum_{k=3}^{n-2}\left(1-x_{k n}^{2}\right)\left|\phi_{k-1}(x)+\phi_{k}(x)\right|\right. \\
&\left.\quad+\sum_{k=3}^{n-2}\left(1-x_{k n}^{2}\right)\left|\phi_{k}(x)+\phi_{k+1}(x)\right|\right\} .
\end{aligned}
$$

Next, we note that for $k=2,3, \ldots, n-2$ (see [13]),

$$
\phi_{k}(x)+\phi_{k+1}(x)=\frac{(-1)^{k} \cos n \theta \sin ^{3} \frac{\pi}{2 n}}{n}\left[I_{k}(\theta)+I_{k}(-\theta)\right]
$$


where

$$
I_{k}(\theta)=\frac{2 \cos \frac{\pi}{2 n}+\cos \left(\theta_{k n}+\theta+\frac{\pi}{2 n}\right)}{\sin \frac{\theta+\theta_{k-1}}{2} \sin \frac{\theta+\theta_{k}}{2} \sin \frac{\theta+\theta_{k+1}}{2} \sin \frac{\theta+\theta_{k+2}}{2}} .
$$

From these observations it follows that

$$
\begin{aligned}
\sum_{k=3}^{n-2}(1 & \left.-x_{k n}^{2}\right)\left|\phi_{k}(x)+\phi_{k+1}(x)\right| \leq\left(1-x^{2}\right) \sum_{k=3}^{n-2}\left|\phi_{k}(x)+\phi_{k+1}(x)\right| \\
& +\sum_{k=3}^{n-2}\left|\left(x^{2}-x_{k n}^{2}\right)\right|\left|\phi_{k}(x)+\phi_{k+1}(x)\right| \\
& \leq C_{4}\left(1-x^{2}\right)+2 \sum_{k=3}^{n-2}\left|x-x_{k}\right|\left|\phi_{k}(x)+\phi_{k+1}(x)\right|
\end{aligned}
$$

By (5.7), (5.8), (5.9) it follows that

$$
\sum_{k=3}^{n-2}\left|x-x_{k}\right|\left|\phi_{k}(x)+\phi_{k+1}(x)\right|=O\left(n^{-2}\right) .
$$

Hence

$$
\sum_{k=3}^{n-2}\left(1-x_{k n}^{2}\right)\left|\phi_{k n}(x)+\phi_{k+1, n}(x)\right|=O\left(1-x^{2}+n^{-2}\right) .
$$

From (5.10) and (5.6) it follows that

$$
|C(x)| \leq \frac{C M_{2}}{4 n^{2}}\left(1-x^{2}+n^{-2}\right) .
$$

Therefore we have

$$
\left|G_{n}\left(f-q_{n-1}, x\right)\right|=O\left(\frac{1-x^{2}}{n^{2}}+\frac{1}{n^{4}}\right) \max _{-1 \leq x \leq 1}\left|f^{\prime \prime}(x)\right| .
$$

(Here we have used (5.2), (5.4), (5.5) and (5.11)). Now from the definition of $H_{n}(f, x)$ as given by (1.10) and (5.12) it follows that

$$
\left|H_{n}\left(f-q_{n-1} x\right)\right|=O\left(\frac{1-x^{2}}{n^{2}}+\frac{1}{n^{4}}\right) \max _{-1 \leq x \leq 1}\left|f^{\prime \prime}(x)\right| .
$$

Let $0 \leq x \leq 1$. We have two cases to consider, First, if $\sqrt{1-x} \geq 1 / n$ then from (5.13) we have

$$
\left|H_{n}\left(f-q_{n-1}, x\right)\right|=O\left(\frac{1-x^{2}}{n^{2}}\right) M_{2} .
$$


Next, consider $\sqrt{1-x}<1 / n$. Since $H_{n}\left(f-q_{n-1}, 1\right)=0$ we have

$$
\begin{aligned}
H_{n}\left(f-q_{n-1}, x\right) & =H_{n}\left(f-q_{n-1}, x\right)-H_{n}\left(f-q_{n-1}, 1\right) \\
& =(1-x) H_{n}^{\prime}\left(f-q_{n-1}, \xi\right), x<\xi<1 .
\end{aligned}
$$

Next, we note that $H_{n}\left(f-q_{n-1}, x\right)$ is itself a polynomial of degree at most $n-1$ and satisfies the inequality (5.13). Therefore, using the Dzyadyk inequality [3] we obtain

$$
\left|H_{n}^{\prime}\left(f-q_{n-1}, x\right)\right|=O\left(\frac{\sqrt{1-x^{2}}}{n}+\frac{1}{n^{2}}\right) M_{2}=O\left(\frac{1}{n^{2}}\right) M_{2} .
$$

Therefore from (5.15) and (5.16) we have

$$
\left|H_{n}\left(f-q_{n-1}, x\right)\right|=O\left(\frac{1-x}{n^{2}} M_{2}\right)=O\left(\frac{1-x^{2}}{n^{2}} M_{2}\right) .
$$

Therefore for $0 \leq x \leq 1$ (similarly also for $-1 \leq x \leq 0$ )

$$
\left|H_{n}\left(f-q_{n-1}, x\right)\right|=O\left(\frac{1-x^{2}}{n^{2}} M_{2}\right) .
$$

This proves the lemma.

\section{Proof of Theorem 1}

Here we will provide the proof of Theorem 1. Let $f \in C^{2}[-1,1]$. Then

$$
\begin{aligned}
f(x) & -H_{n}[f, x] \\
& =f(x)-q_{n-1}(x)+q_{n-1}(x)-H_{n}\left[q_{n-1}, x\right]+H_{n}\left[q_{n-1}-f, x\right]
\end{aligned}
$$

where $q_{n-1}(x)$ is the polynomial of degree at most $n-1$ satisfying (4.1). By (4.1), (4.9) and (5.1) we have

$$
\left|f(x)-H_{n}[f(x)]\right|=0\left(\frac{1-x^{2}}{n^{2}}\right) \max _{-1 \leq x \leq 1}\left|f^{\prime \prime}(x)\right| .
$$

Now applying the theorem of Ron DeVore [5] (Theorem [2.4]) it follows that if $f \in C[-1,1]$ then

$$
\mid f(x)-H_{n}\left(f(x) \mid \leq C \omega_{2}\left(f, \frac{\sqrt{1-x^{2}}}{n}\right) .\right.
$$

This proves our main theorem. 


\section{Proof of Theorem 2}

Here we provide the details of the proof of Theorem 2. Let $f \in C^{(2)}[-1,1]$. First we note that

$$
\begin{aligned}
f^{\prime}(x)-H_{n}^{\prime}(f, x)= & f^{\prime}(x)-P_{n-1}^{\prime}(x)+P_{n-1}^{\prime}(x)-H_{n}^{\prime}\left(P_{n-1}, x\right) \\
& +H_{n}^{\prime}\left(P_{n-1}, x\right)-H_{n}^{\prime}(f, x) \\
= & J_{1}+J_{2}+J_{3} .
\end{aligned}
$$

Here we have chosen $P_{n-1}(x)$ to be the approximating polynomial of $f(x)$ on $[-1,1]$ such that

$$
\left|f(x)-P_{n-1}(x)\right| \leq C_{5}\left(\frac{1-x^{2}}{n^{2}}+\frac{1}{n^{4}}\right) M_{2}, M_{2}=\max _{-1 \leq x \leq 1}\left|f^{\prime \prime}(x)\right|
$$

and

$$
\left|f^{\prime}(x)-P_{n-1}^{\prime}(x)\right| \leq C_{6}\left(\frac{\sqrt{1-x^{2}}}{n}+\frac{1}{n^{2}}\right) M_{2} .
$$

The existence of such a polynomial is well known (see [11, Theorem 1, p. 164]). Clearly

$$
\left.\left|J_{1}\right|=\mid f^{\prime}(x)-P_{n-1}^{\prime}(x)\right) \mid=O\left(\frac{\sqrt{1-x^{2}}}{n}+\frac{1}{n^{2}}\right) M_{2} .
$$

Following as in the proof of (4.2) it follows that

$$
\left|P_{n-1}(x)-H_{n}\left(P_{n-1} x\right)\right|=O\left(\frac{1-x^{2}}{n^{2}}+\frac{1}{n^{4}}\right) M_{2} .
$$

Now, we note that $P_{n-1}(x)-H_{n}\left(P_{n-1}, x\right)$ is itself a polynomial of degree at most $n-1$ satisfying (7.5) and from Dzyadyk inequality [3] we obtain

$$
\left|P_{n-1}^{\prime}(x)-H_{n}^{\prime}\left(P_{n-1}, x\right)\right|=C_{7}\left(\frac{\sqrt{1-x^{2}}}{n}+\frac{1}{n^{2}}\right)\left\|f^{\prime \prime}\right\| .
$$

Similarly we also obtain for $f \in C^{2}[-1,1]$

$$
\left|H_{n}\left(P_{n}-f, x\right)\right| \leq C_{8}\left(\frac{1-x^{2}}{n^{2}}+\frac{1}{n^{4}}\right) M_{2},-1 \leq x \leq 1
$$

and

$$
\left|H_{n}^{\prime}\left(P_{n-1}-f, x\right)\right| \leq C_{9}\left(\frac{\sqrt{1-x^{2}}}{n}+\frac{1}{n^{2}}\right) M_{2},-1 \leq x \leq 1
$$


Therefore for $f \in C^{(2)}[-1,1]$ we obtain from (7.1), (7.4), (7.6) and

$$
\left|f^{\prime}(x)-H_{n}^{\prime}(f, x)\right| \leq C_{10}\left(\frac{\sqrt{1-x^{2}}}{n}+\frac{1}{n^{2}}\right) M_{2},-1 \leq x \leq 1 .
$$

Therefore applying DeVore [Theorem 2.5] we have for $f^{\prime} \in C[-1,1]$

$$
\left|f^{\prime}(x)-H_{n}^{\prime}(f, x)\right| \leq C_{11} \omega\left(f^{\prime}, \frac{\sqrt{1-x^{2}}}{n}+\frac{1}{n^{2}}\right) .
$$

The proof of (1.14) is easy and so we omit the details.

\section{Proof of Theorem 3}

Proof of Theorem 3. From Theorem 2, we have

$$
\left|H_{n}^{\prime}(f, \pm 1)-f^{\prime}( \pm 1)\right| \leq C \omega\left(f^{\prime}, 1 / n^{2}\right) .
$$

Also, we note that

$$
\left|\frac{T_{n}(x)-T_{n}(1)}{n^{2}}\right| \leq \frac{2}{n^{2}},\left|\frac{T_{n}(x)-T_{n}(1)}{n^{2}}\right| \leq 1-x .
$$

Combining (8.1) and (8.2), we obtain

$$
\begin{aligned}
\left|I_{1}(x)\right|: & =\left|\frac{T_{n}(x)-T_{n}(1)}{n^{2}}\right|(1+x)^{2}\left[H_{n}^{\prime}(f, 1)-f^{\prime}(1)\right] \mid \\
& \left.\leq C \min \left\{1 / n^{2}, 1-x, 1+x\right)\right\} \omega\left(f^{\prime}, 1 / n^{2}\right) .
\end{aligned}
$$

If $1-x^{2} \geq 1 / n^{2}$, then we have

$$
\left|I_{1}(x)\right| \leq C \frac{1}{n^{2}} \omega\left(f^{\prime}, \frac{1}{n^{2}}\right) \leq C \frac{\sqrt{1-x^{2}}}{n} \omega\left(f^{\prime}, \frac{\sqrt{1-x^{2}}}{n}\right) .
$$

If $0<1-x^{2}<1 / n^{2}$, we have $1-x^{2} \leq \frac{\sqrt{1-x^{2}}}{n}$,

$$
\left|I_{1}(x)\right| \leq C\left(1-x^{2}\right) \omega\left(f^{\prime}, \frac{1}{n^{2}}\right) \leq C \frac{\sqrt{1-x^{2}}}{n} \omega\left(f^{\prime}, \frac{\sqrt{1-x^{2}}}{n}\right) .
$$

Moreover, we have $I_{1}( \pm 1)=0$. Hence for $x \in[-1,1]$ we obtain

$$
\left|I_{1}(x)\right| \leq C \frac{\sqrt{1-x^{2}}}{n} \omega\left(f^{\prime}, \frac{\sqrt{1-x^{2}}}{n}\right) .
$$


Similarly we have

$$
\begin{aligned}
\left|I_{2}(x)\right| & :=\left|\frac{T_{n}(x)-T_{n}(-1)}{T_{n}^{\prime}(1)}(1-x)^{2}\left(H_{n}^{\prime}(f,-1)-f^{\prime}(-1)\right]\right| \\
& \leq C \frac{\sqrt{1-x^{2}}}{n} \omega\left(f^{\prime}, \frac{\sqrt{1-x^{2}}}{n}\right) .
\end{aligned}
$$

Thus, from Theorem 1, (8.3), (8.4) and (1.15), we get

$$
\left|f(x)-J_{n}(f, x)\right| \leq C \frac{\sqrt{1-x^{2}}}{n} \omega\left(f^{\prime}, \frac{\sqrt{1-x^{2}}}{n}\right) .
$$

By (8.1) and the definition of $I_{1}(x)$ we have

$$
\left|I_{1}^{\prime}(x)\right| \leq C \omega\left(f^{\prime}, \frac{1}{n^{2}}\right),\left|I_{2}^{\prime}(x)\right| \leq C \omega\left(f^{\prime}, \frac{1}{n^{2}}\right) .
$$

Then from Theorem 2, and the above estimates

$$
\left|f^{\prime}(x)-J_{n}^{\prime}(f, x)\right| \leq C \omega\left(f^{\prime}, \frac{\sqrt{1-x^{2}}}{n}+\frac{1}{n^{2}}\right) .
$$

On the other hand it is easy to verify that $f^{\prime}( \pm 1)-J_{n}^{\prime}(f, \pm 1)=0$. Hence, using similar arguments as before, we can prove that

$$
\left|f^{\prime}(x)-J_{n}^{\prime}(f, x)\right| \leq C \omega\left(f^{\prime}, \frac{\sqrt{1-x^{2}}}{n}\right) .
$$

This proves Theorem 3 as well.

\section{References}

[1] Ju. A. Brudnyi, 'Generalizations of a theorem of A. F. Timan', Soviet Math. Dokl. 4 (1963), 244-247.

[2] V. K. Dzyadyk, 'A further strengthening of Jackson's theorem on the approximation of a continuous functions by ordinary polynomials', Dokl. Akad. Nauk 121 (1958), 403-406.

[3] V. K. Dzyadyk, 'On a constructive characteristic of functions satisfying the Lipschitz condition on a finite segment of the real axis', Izv. Akad. Nauk, SSR 20 (1956), 623-642.

[4] R. DeVore, 'Degree of approximation', Approximation Theory II, edited by Lorentz, Chui, Schumaker, pp. 117-162 (Academic Press, 1976).

[5] R. DeVore, 'Pointwise approximation by polynomials and splines', Proceedings of the conference on the constructive function theory, pp. 132-141 (Kalouga, Soviet Union, 1977).

[6] G. G. Lorentz, Approximation of Functions, 2nd ed. (Chelsea, NY, 1986). 
[7] X. Ming Yu, 'Pointwise estimate for algebraic polynomial approximation', Approx. Theory and Applications, 1 (3) (1985), 109-115.

[8] A. K. Varma and T. M. Mills, 'A new proof of Telyakovski's Theorem on approximation of functions', Studia Sci. Math. Hungar. 14 (1979), 241-256.

[9] A. K. Varma and T. M. Mills, 'A new proof of Timan's Approximation theorem', Israel J. Math. (1974), 39-44.

[10] S. M. Nikolskii, 'On the best approximation of functions satisfying Lipschitz's conditions by polynomials', Izv. Akad. Nauk. SSR 77 (1951), 969-972.

[12] A. F. Timan, 'Strengthening of Jackson's theorem on the best approximation of continuous functions on a finite segment of the real axis', Dokl. Akad. Nauk. 78 (1951), 17-20.

[13] A. K. Varma, 'A new proof of A. F. Timans Approximation Theorem II', J. Approx. Theory, 18 (1) (1976), 57-62.

University of Florida

Gainesville, Florida 32611

U.S.A.
University of South Carolina

Columbia, South Carolina 29208

U.S.A. 